\title{
Experimentelle Untersuchungen über die Tiefenwirkung der Kromayer'schen Quarzlampe (Quecksilberdampflicht) an normaler Haut.
}

Von

\section{Dr. Rudolf Pürckhauer,}

Assistenten der Klinik.

Die in letzter Zeit von verschiedenen Seiten mitgeteilten Heilerfolge mittels der $\mathrm{Kr}$ omayerschen Quarzlampe haben mich veranlaßt, eine Prüfung der Lichtreaktion an normaler Haut vorzunehmen. Bisher hat sich meines Wissens noch keine Arbeit mit den histologischen Veränderungen, die durch die Quarzlampe im lebenden Gewebe gesetzt werden, beschäftigt. Aber nur auf diese Weise läßt sich ein Urteil fällen über die für die Therapie verwertbare Tiefenwirkung einer Lichtquelle. Die absolute Tiefenwirkung des Lichtes im lebenden Gewebe nach dem Ausschlag zu bemessen, den das Licht nach Durchdringung tierischer Membranen auf lichtempfindliche Substanzen gibt, ist schon deswegen nichtzulässig, weil hierdurch nicht der Beweis erbracht ist, da $\beta$ der belichtete lebende Organismus a ch wirklich vonden Strahlen nachhaltig beeinflußt wird. Ich habe nun ähnlich, wie dies von Veress, ${ }^{1}$ ) $\mathrm{Z}$ i el er, ${ }^{2}$ )

1) F. von Veress: Über die Wirkung des I'insenlichtes auf normale Haut. Monatsschrift für praktische Dermatologie. 1905, Bd. XL.

2) K. Zieler: Über die Wirkung des konzentrierten elektrischen Bogenlichtes nach Finsen auf die normale Haut. Derm. Zeitschr. Bd. XIII. Heft 1, 1906. 
Jansen ${ }^{1}$ ) bei dem Finsen licht klargelegt haben, die Lichtwirkung der Quarzlampe am Kaninchenohr studiert. Es wurden zu diesen Experimenten ausschließlich ausgewachsene Albinokaninchen verwandt. Die zu belichtende Innenfläche des Ohres wurde vorsichtig rasiert und mit Benzin entfettet. Mit einem Druckglas wurde die Innenfläche sodinn an das Quarzfenster, welches mit Zinnfolie bis auf eine zweimarkstückgroße Fläche $a b-$ gedeckt war, aufgepreßt, so daß das $\mathrm{Ohr}$ im bestrahlten Bezirk möglichst anämisiert wurde. In dieser Lage erfolgte eine einstündige ununterbrochene Belichtung des Ohres, wobei dem Küblwasser der Lampe bei der Hälfte der Versuche Methylenblau in einer Konzentration von 1:7500 beigefügt wurde, um so - nach Augabe K rom a yers ${ }^{2}$ ) - die ganz oberflächlich wirkenden kurzwelligen, ultravioletten Strahlen möglichst auszuschalten. Diese Versuchsanordnung wählte ich, weil nach Kromayers Angabe eine einstündige Bestrablung mit der Quarzlampe der gewöhnlichen $5 / 4$ stündigen Bestrablung mit der Finsenlampe mindestens gleichwertig ist. Auf diese Weise wurden Ergebnisse gewonnen, die mit den Befunden der erwähnten Untersucher vergleichbar sind. - Ich möchte hier erwähnen, daß bei einigen Versuchen eine völlige Anämie deswegen nicht erzielt werden konnte, weil das Tier unruhig wurde und das aufgelegte Druckglas sich etwas lockerte. Bei diesen nicht einwandsfreien Versuchen, die im folgenden nicht berücksichtigt wurden, trat die Lichtreaktion, bei der jedenfalls eine Wärmewirkung mitspielte, viel rascher als gewöhnlich ein.

Die Belichtung wurde ausgeführt bei einer Stromstärke von $3 \frac{1}{2}$ Amp. und einer Spannung von 110 Volt. Nach Ablauf verschieden langer Zeitabschnitte wurde das belichtete Ohr abgeschnitten und zur histologischen Untersuchung teils in Z enkerscher Flüssigkeit, teils in Formalin fixiert. Die Färbung der Paraffinschnitte erfolgte nach W e ig e r t-van Gi e s on,

1) Jansen: Über Gewebssterilisation und Gewebsreaktion bei Finsens Lichtbehandlung. Zieglers Beiträge z. path. Anatomie a. allg. Path. 1907, Bd. 41, pag. 277.

2) Kromayer: Die Anwendung des Lichtes in der Dermatologie. Berliner klinische Wochenschrift 1907. Nr. 4 u. 5. 
Giemsa, May-Grünwald, Zieler, Heidenhain, mit Hämatoxylin-Eosin, polychromen Methylenblau, LithionkarminBismarckbraun und Weiger tscher Farblösang tür elastische Fasern.

\section{Makroskopische Veränderungen am bestrahlten $0 \mathrm{hr}$.}

Unmittelbar nach einstündiger Bestrahlung kann nichts Besonderes wahrgenommen werden. Nach vier Stunden erscheinen die Gefäße weit, mit Blut gefüllt. Nach 12 Stunden tritt eine Hyperämie auf, die nach 24 Stunden an Deutlichkeit zunimmt. Der bestrahlte Bezirk füblt sich warm an und erscheint gerötet. Bis zu diesem Zeitpunkt besteht ein wesentlicher Unterschied in der Reaktion zwischen Bestrahlung mit und ohne Methylenblau nicht, Erst nach dieser Zeit tritt eine Differenz ein.

Mit Methylenblan: Nach drei Tagen erscheinen in der Peripherie kleine Blutungen; die stärker gerötete, bestrahlte Stelle schuppt leicht und ist verdickt. Nach fünt Tagen erkennt man eine zarte Bläschenbildung, besonders am Rand der geröteten Stelle, die jetzt erheblich verdickt ist. Die Exsudation nimmt nun von Tag zu Tag zu und erreicht nach acht bis zehn Tagen ihren Höhepunkt, indem sich eine derbe dicke Kruste gebildet hat. Von dieser Zeit löst sich der Schorf zunächst an der Peripherie und fällt nach zwei bis drei Wochen spontan ganz ab. Nach vier bis fünf Wochen bestehen äußerlich wieder normale Verhältnisse, abgesehen von einer fühlbaren Verdickung innerhalb der bestrablten Stelle.

Ohne Methylenblau: Nach drei Tagen erscheint eine deutliche Exsudation, die in der Peripherie beginnend, nach fünf Tagen sich am gesamten bestrahlten Bezirk, der stark verdickt ist, bemerkbar macht. Peripher ist das Extravasat eingetrocknet und bildet hier ein Börkchen. Nach einer Woche ist die belichtete Stelle von einer dicken festhaftenden Kruste bedeckt. Diese beginnt sich erst nach Verlauf von zwei bis drei Wochen am Rande zu lösen. Nach 14 Tagen beobachtet man an der unbestrahlten Seite eine deutliche Schuppung der obersten Hautschicht. Die Haare fallen hier aus. Nach vier Wochen ist die Kruste spontan abgefallen und es erscheint darunter eine völlig regenerierte, etwas hyperämische Haut. Als Residuen der Lichtwirkung gewahrt man eine Faltenbildung des verdickten Gewebes, wenn nicht, wie ich dies einigemal beobachtete, durch die resultierende Gewebsnekrose ein Defekt in Gestalt eines stecknadelkopfgroßen oder größeren Loches gesetzt wird.

Diese makroskopische Lichtreaktion stimmt bei allen Versuchen im wesentlichen überein.

\section{Mikroskopische Untersuchung.}

Zum histologischen Befund am normalen Kaninchenohr möchte ich nur kurz folgendes erwähnen: Innen- und Außenfläche zeigen einen 
dünnen Epithelsaum, der an der Außenseite um eine Zellschicht (zirka 4) breiter ist. Man erkennt eine Hornschicht und ein Rete Malpighi. Ein Stratum papillare existiert nicht. Haarbälge und Talgdrüsen finden sich beiderseits, jedoch häufiger und stärker entwickelt an der Außenfläche. Die Tela subcutanea, wenn man eine solche abtrennen will, beherbergt die größeren Blut- und Lymphgefäße sowie Nerven. Der beide Seiten trennende elastische Knorpel wird beiderseits von einem zarten Perichondrium umschlossen.

\section{Belichtung ohne Zusatz von Methylenblau.}

1. Nach einer Stunde: Fast keine Veränderung. Cutis und Subcutis auf der abgewandten Seite etwas aufgelockert, Gefäße blutreich, weit. Epithelien und Gefäßendothelien unbeeinflußt.

2. Nach vier Stunden: Am Epithel und den Zellen der Haarbälge und Talgdrüsen sind noch keine Veränderungen aufgetreten, außer geringer Abblassung der Kerne. Die Gefäße der kaum aufgelockerten Cutis sind prall gefüllt. Ein ganz geringes Ödem macht sich kenntlich durch ein flockiges fibrinöses Exsudat sowie durch extravasierte Zellen (Lymphound Leukocyten): Hier und da beobachtet man einige rote Blutkörperchen frei im Gewebe. Die nicht belichtete Außenfläche des Ohres ist leicht gequollen, die einzelnen Bindegewebsfibrillen auseinander gedrängt.

3. Nach zwölf Stunden: Das Zellexsudat ist reichlicher geworden, man beobachtet poly- und mononucleäre, nentrophile und einzelne eosino- und basophile Zellen zwischen den Spalträumen und geringe Fibrinausscheidung. Das Epithel ist auf der bestrahlten Seite mangelhaft gefärbt, die diapedetischen Blutungen noch gering. Das Ödem und die Fibrinausscheidung treten stärker auf der unbestrahlten Seite hervor.

4. Nach ei nem Tag: Die Färbbarkeit der Kerne der Epidermiszellen hat stark gelitten, die Zellen erscheinen nicht mehr scharf gegen einander abgesetzt. Talgdrüsenläppehen und Haarbälge sind vielfach ebenso geschädigt. Die kleineren oberflächlichen Blutgefäße haben z. T. ihre Endothelien abgestoBen und sind thrombosiert. In der Tiefe ist nichts von einer Thrombose wahrnehmbar. Ein deutlicher Kernzerfall macht sich in den oberen Cutisschichten bemerkbar, besonders in den Gefäßwandzellen, überall also ausgesprochene Zeichen der Nekrose: Diapedese roter Blutkörperchen sieht man vereinzelt auftreten. Der Knorpel zeigt keine Veränderung. Die Bindegewebsfasern sind auf der unbestrahlten Seite von Exsudatzellmassen und ziemlich bedeutenden Fibrinmengen auseinander gedrängt. Dieses ödem ist jedoch a uf der belichteten Seite sehr wenig und nur in den tiefsten Schichten bemerkbar. Von Proliferationsvorgängen ist nirgends etwas zu bemerken, auch nicht auf der abgewandten Seite. 
5. Nach drei Tagen: In diesem Stadium findet man bereits eine völlige Nekrose des Epithels, die sich auch tiefer in der Cutis bemerkbar macht. Sämtliche Zellelemente sind an diesen Stellen zerfallen, die Gefäße thrombosiert; auch die Talgdrüsen und Haarbälge teilweise nekrotisch. Die tiefen Gefäße sind zumeist noch frei von Gerinnungen, doch zeigen sie Schädigungen der Gefäßwand (mangelhafte Kernfärbung der Endothelien). Überall im Gewebe verstreut liegen zahlreiche Leukocyten und besonders Erythrocyten. $Z$ wischen den Bindegewebsfasern der Cutiserkenntmanmäßige Fibrinausschwitzung a uf der bestrahlten Seite. Hinter dem unveränderten Krorpel zeigt sich ein breiter Saum dicht gedrängter Leukocyten, die an den Stellen, wo der Knorpel Lücken hat, in breitem Schwarm nach der belichteten Seite hinüberwandern (Chemotaxis). Die Zellelemente der unbelichteten Seite sind wenig beeinträchtigt, die Auflockerung der Cutis ist stärker geworden, aber im ganzen nicht so bedeutend als bei Bestrahlung mit Methylenblau. Eine Zellneubildung ist immer noch nicht im Gang.

6. $\mathrm{Nach}$ einer Woche: Die Reaktion ist weiter vorgeschritten. Das Epithel und dessen Anhangsgebilde sind im bestrahlten Bezirk einer totalen Nekrose anheimgefallen. Kernfärbung wird ganz vermißt. Die Gefäße des Coriums sind völlig thrombosiert, die der Subcutis zum größten Teil mit Blutkörperchen prall angefüllt, wenn nicht gleichfalls im Stadium der Thrombose. Rote Blutkörperchen liegen in reichlicher Menge im Gewebe. Unter dém Knorpel hat die Leukocytenansammlung an Menge noch zugenommen. Der Knorpel ist schlecht gefärbt. In den Blutgefä円en der Außenseite besteht vielfach eine Stase, dagegen beobachtet man nirgends Thrombenbildung. Hier beginnt jetzt eine Vermehrung der Bindegewebszellen besonders in der Umgebung der Gefäße und der Gefäßwandzellen. Das Epithel ist bedeutend hypertrophiert, etwa um das Dreifachedes Normalen. Ebenso wie sich hier eine deatliche entzündliche Zellneubildung zeigt, beginnt auch an den Randpartien derbelichteten Seite eine Wucherung des Epithels, der Haarbälge und Drüsen. Im w uchernden Bindegewebe erscheinen dieverschiedensten mononucleären Rundzellen, Leukocyten und Mastzellen. Im Zentrum der Bestrahlung sind regenerative Vorgänge auch in der Tiefe noch kaum angedeutet.

7. Nach vierzehn Tagen: Die Nekrose im bestrahlten Bezirk ist bis auf den Knorpel vorgeschritten, die Zellgrenzen höchstens noch angedeutet, die Kerne total zerfallen. Die tiefen Gefäße sind mit starren Thromben gefüllt. Das nekrotische Gewebe ist von Lympho- und Leukocyten und Resten roter Blatkörperchen durchsetzt. Im Zentrum des bestrahlten Gebietes erscheint der Knorpel an der Innenfläehe wie aufgefasert. Die Knorpelzellen sind nicht gefärbt, das Perichondrium stark aufgelockert. Neben diesen zentral sich abspielenden degenerativen Er- 
scheinungen beobachtet man an den Randpartien ein kräftiges, im Entstehen begriffenes Granulationsgewebe: Junge Fibroblasten mit länglichem spindelförmigen Zelleib, zuweilen mit langen Fortsätzen versehen, Leukocyten mit polymorphem Kern, einkernige Rundzellen, eosinophile und Mastzellen; dazwischen junge wuchernde Gefäßendothelien. Ein breiter hypertrophischer Epithelsaum schiebt sich vom Rand nach den nekrotischen Partien vor und unterwächst die Nekrose. Die Drüsen an der Peripherie stark gewuchert. Dieselben Wucherungsprozesse, wenn auch nicht in so ausgedehntem Maße, zeigen sich auf der unbelichteten Seite. Der Leukocytenwall hinter dem Knorpel hat noch um ein Bedeutendes zugenommen und bildet gleichsam eine Demarkationslinie.

8. Nach drei Wochen: Während in den Randpartien der bestrahlten Stelle eine lebhafte entzündliche Gewebsneubildung erkennbar ist, hat im Zentrum die Nekrose ihren Höhepunkt erreicht. Der Knorpel ist in toto abgestorben, nur die Zellgrenzen sind eben roch erkennbar. Das Zenirum des bestrahlten Gewebes bildet eine nekrotische Masse mit Kerntrümmern, Fil.rinmengen, Leukocyten etc. Selbst die elastischen Elemente sind vielfach untergegangen. Auch auf die unbelichtete Seite haben die regressiven Prozesse übergegriffers. Epitheliale und CutisElemente sind mehr oder weniger der Nekrose verfallen. An der Peripherie der belichteten Stelle ist die Zellproliferation eine ganz bedeutende. Die Epithelüberhäutung schreitet vom Rand her mächtig vor. Dabei ist das Epithel häufig in atypische Wucherungen geraten, indem es Zapfen und Stränge in das breite Granulationsgewebe hineinschickt. An einzelnen Stellen sind die Wucherungen derartig stark, daf sie bis in den Knorpel eindringen und hier die nekrotischen Knorpelelemente ersetzen. Auf der unbestrablten Seite am Rand der Knorpelnekrose beginnt eine Knorpelneubildung.

9. Nach sieben Wochen: Die Überhäutung ist jetzt völlig abgeschlossen. Das Epithel ist noch hypertrophisch, doch ist die Rückbildung deutlich ausgeprägt. Man sieht im Gewebe keine degenerativen Prozesse mehr, wenn man Abstand nimmt von den Vorgängen im Knorpel selbst. Dagegen bestehen in der Subcutis und besonders in der Cutis noch immer lebhafte entzündliche Neubildungsprozesse, kenntlich an den zahlreichen Kernteilungsfiguren und den unfertigen Formen der Bindegewebselemente, sowie an den reichlichen jungen Gefäßsprossen. Riesenzellen scheinen nicht aufzutreten. Haarbälge und Drüsen sind auf der belichteten Seite nur wenig regeneriert. Was den Knorpel selbst betrifft, so ist dieser im bestrablten Bezirk völligwiederhergestellt. Die nekrotischen Knorpelmassen sind auf beiden Seiten von jungem Knorpelgewebe umwuchert. Die neugebildeten Knorpelzellen sind zarter, viel kleiner und haben keine Zelleinschlüsse. In der jungen Knorpelgrundsubstanz sind schon feinste elastische Fasern vorhanden, z. T. in sehr dichter Anordnung, allerdings nicht so dicht wie im alten Knorpel, aber 
in derselben Lagerung. Die Neubildung der elastischen Fasern geht also hier außerordentlich schnell vorsich. Die Hyperplasie des Knorpelgewebes beschränkt sich jedoch nicht a uf die bestrahlte. Stelle allein, sondernsie macht sichauch weiterhin am unbelichteten Knorpel etwas bemerkbar. Hier siebt man wie die alten Knorpelzellen von den jungen umschlosen werden. Während die alten Zellen sich durchibre GröBe und durch diemangelbaftebis ganzfehlendeKernfärbung hervorheben und weit a useinanderliegen, setzen sich die jungen Zellen scharf ab durch ibre deutliche Protoplasma- und Kernfärbung. Viele junge Knorpelzellen, deren Gestaltsehr variabelist, drängen sich zwischendie alten, vonder Belichtung geschädigten ein and bringensie vollends zum Untergang. Durch die das zu GrundegegangeneGewebe weit übertreffende Neubildung des Knorpels entstehen. ganz eigenartige Bilder. Die sonst gerade und gleichmäbig verlaufende dünne Lamelle des normalen Knorpels zeigt Verbreiterungen und UnregelmäBigkeiten und übertrifft dienormale Dickeum das Vierbis Fünfache. Diese Knorpelzellenneubidung geht vomerhaltengebliebenen Perichondrium aus. Auf der unbestrahlten Seite ist das Gewebe annähernd normal, abgesehen von einer Hyperplasie sämtlicher Bestandteile in toto.

\section{Mit Zusatz von Methylenblau.}

1. N a ch einer Stunde: Im ganzen ist das histologische Bild wenig beeinflußt. Das Gewebe ist wenig aufgelockert. Lie Gefäße erweitert, mit Blut gefüllt.

2. $\mathrm{Na}$ c h vier $\mathrm{S} t$ un de $\mathrm{n}$ : Innerhalb der Epithelzellen beobachtet man vereinzelte Vacuolen, jedoch hat die Färbbarkeit der Kerne nicht wesentlich.gelitten. In den tiefen Schichten zeigt sich frei im gelockerten Gewebe eine Fibrinausschwitzung in Gestalt eines zarten Fasernetzes. Die Gefäße sind z. T. voll gepfropft von Blutkörperchen, deren Struktur bisweilen undeutlicb ist. An solchen Gefäßen tritt eine beginnende Diapedese auf. Die normaliter sehr engen Lymphräume sind stark erweitert, einzelne mit fibrinösen Massen erfülit.

3. Nach zwölf Stunden: Die Zellkerne der bestrahlten Epidermis sind gequollen, besser gefärbt als ohne Methylenblaubestrahlung, aber weniger als auf der unbelichteten Seite. Die Cutis und Subcutis ist ödematös a ufgelockert, die Bindegewebsbündel auseinander gedrängt. In den so entstandenen Maschen finden sich reichliche Fibrinmengen und Exsudatzellen, unter denen zahlreiche pseudoeosino- 
phile und nur vereinzelt eosinophile Zellen auftreten. Di e A u Benfläche ist weit stärkerödematös gequollen undenthält viel mehrFibrinmengen in ihrenGewebsmaschen als die bestrablte Seite.

4. Nach e in em Tag: Die Epidermiszellen sind wenig mehr gequollen, Kerne z. T. schlecht tingiert. Die Haarbälge sind im allgemeinen ohne Zeichen beginnender Nekrose. In den Bindegewebsschichten hat die Zellexsudation zugenommen. Rundzellen, Leukocyten, Erythrocyten liegen in reichlicher Menge frei in dem gelockerten Gewebe. Vereinzelte oberflächliche Gefäße sind thrombosiert. Knorpel ohne Veränderung, dagegen erscheint das Perichondrium beiderseits verbreitert (aufgelockert). An der Knorpelaußenfläche beginnt eine Leukocytenansammlung. Auf der nicht belichteten Seite sind im Gegensatz zur bestrahlten Fläche die einzelnen Fibrillen des Bindegewebes außerordentlich stark auseinander gedrängt und die $Z$ wischenräume mit Exsudatmassen erfüllt (ganz ähnlich wie bei Finsenbestrahlung).

5. Nach drei Tagen: Am Epithel selbst ist noch wenig zu erkennen, es erscheinen die Zellen heller, die Kernfärbung herabgesetzt. Irgendwelche Blasenbildungen bestehen nicht. Die oberflächlichen Gefäße sind zum großen Teil thrombosiert, die tiefen noch immer thrombenfrei, doch mehr oder weniger mit Blutelementen ausgefüllt. Die exsudierten Zellen, insbesondere die sogenannten lymphocytären Elemente haben an Zahl, besonders an der unbestrablten Außenfläche zugenommen. Die am meisten hervorspringende Veränderung ist $n a c h$ wie vor die besonders stark a ufderunbestrablten Seite auftretende Fibrinausschwitzung, die in Form von feinsten Fasernetzen die Zellzwischenränme mehr oder weniger ausfüllt. An der nichtbelichteten Seite findet man Kernteilungen jn Bindegewebszellen, nur ganzvereinzeltein Gefäßwandzellen. An den Randpartien tritt deutlich eine Epithelwucherung zu Tage.

6. Nach einer Woche: Das bestrahlte Epithel ist in toto nekrotisch. Ein dicker aus Zelltrümmern, Fibrinmassen und Leukocyten bestehender Schorf lagert an der Stelle des Epithels. Die Nekrose erstreckt sich weiter in die Tiefe, so ist von Haarbälgen und Talgdrüsen fast nichts mehr erkennbar. Überhaupt sind die epithelialen Elemente besonders stark geschädigt, meistens nekrotisch. Nur einzelne grölere Gefäße in der Tiefe lassen ihre Struktur eben erkennen, doch sind die Kerne der Gefäßwandzellen zerfallen. In Cutis und Subcutis der peripheren Abschnitte spielt sich jetzt eine lebhafte entzündliche Neubildung ab. Junge Fibroblasten schieben sich zwischen die erbaltenen Bindegewebsbündel hinein. A u ch in der Tiefeder bestrab!ten Partie beginnt bereit s eine Zellproliferation. Die Endothelien der GefäBe sind in starker Wucherung begriffen, kenntlich an den großen gequollenen Kernen. Allenthalben trifft man reichlich Kernteilungsfiguren. An den Randpartien ist das Epithel, die Zellen der Haarbälge und Talgdrüsen stark hyper- 
trophiert. Die Knorpelzellen sind schlecht gefärbt. Die unbelichtete Fläche befindet sich gleichfalls in energischer Regeneration. Man beobachtet in Epithel-, Bindegewebs- und Gefäßwandzellen die verschiedensten Kernteilungen.

7. Nach vierzehn Tagen: Ein dicker Schorf von Fibrinmengen, Hornmassen und Leukocyten hat sich abgestoßen und steht nicht mehr in Verbindung mit der Haut. Unter diesem Schorf hat sich das E $\mathrm{p}$ ithel zum größten Teil regeneriert. Die Epithelüberhäutung schreitet von den Randpartien, woselbst die Epithelzellen der Epidermis in starker Proliferation begriffen sind (zirka 10 Zellschichten) nach dem Belichtungszentrum vor. An Stelle des nekrotischen Bindegewebes sind junge Gewebselemente getreten. GroBe Mengen spindelförmiger Fibroblasten mit groben gequollenen Kernen, zarte GefäBkapillaren und Rundzellen bilden das Granulationsgewebe, in welchem sich die verschiedensten Kernteilungsbilder zeigen. Die lebhafteste Wucherung findet man im Bindegewebe in der Tiefe der bestrahlten Seite. Die progressiven Yorgänge an der Außenseite haben etwas nachgelassen. Hier ist der gesamate Epithel sehr stark hypertrophiert und hat papillenähnliche Zapfen in die Cutis hineingeschickt. Besonders das normal fast ganz fehlende Stratum granulosum hat eine ganz erhebliche Breite gewonnen. Im Knorpel erscheinen die Kerne schlecht oder bisweilen gar nicht tingiert.

8. Nacb drei Wochen: Vonden degenerativen Vorgängen ist fast nichts mehr zu sehen. Das Epithel ist fast völlig. regeneriert, in Zapfen und Strängen gewuchert. Die Keratohyalinschicht und das Rete Malpighi haben je bis sechs Zellschichten; die Zellen sind grob und protoplasmareich, ihre Kerne stark gefärbt. Ein junges Granulationsgewebe hat sich in Cutis und Subcutis ausgebreitet, doch scheinen die entzündlichen Neubildungsvorgänge in der Tiefe mehr zum Stillstand gekommen za sein. Man findet hier weniger Teilungsfiguren, die neugebildeten Bindegewebszellen haben eine regelmäßige Anordnung erlangt, indem sie sich dem Verlauf der alten nicht der Nekrose verfallenen Bindegewebsfasern angepaßt haben. Dagegen ist das Granulationsgewebe unterhalb der Epidermis noch ohne jede Struktur, die Zellen sind in lebhafter Teilung begriffen. Eine sehr wichtige regenerative Veränderung findet man an mehreren Stellen der Knorpelaußenfläche, indem sich hier vom Perichondrium ausgehend neue Knorpelzellen gebildet haben. Sie unterscheiden sich von den übrigen Knorpelzellen durch ihre zarten Kapseln, die Kleinheit der Kerne und dadurch, daß sie nie Zelleinschlüsse enthalten. Die elastischen Fasernetze sind bereits im neugebildeten Knorpelgewebe vorhanden.

9. Nach fünf Wochen: Das Epithel zeigt etwa fünf bis sechs Zellreihen. Die Zellen haben normale Größe und Beschaffenheit. Die entzündliche Neubildung in Cutis und Subcutis hat nachgelassen. Haar- 
bälge und Talgdrüsen sind nur äußerst spärlich regeneriert. Auf der belichteten Seite ist die Wandung der größeren Gefäße beträchtlich verdickt. Man beobachtet in der großen Arterie eine gewalfige Hyperplasie der Intimazellen, wodurch das Lumen eingeengt wird. Der Knorpel ist im Zentrum der belichteten Stelle völlig neugebildet und hat an Breite etwa um das fünffache des Normalen zugenommen. In den jungen Zellen zahlreiche Kernteilungsfiguren. Die Art der Knorpelneubildungen ist die gleiche wie sie oben bei der. Bestrahlung ohne Methylenblau (nach sieben Wochen) beschrieben worden ist. Die Verbältnisse auf der Außenseite sind ungefähr wieder normale, nur ist eine mächtige Hyperplasie in allen Gewebsschichten eingetreten.

Im Anschluß an diese meine Versuche sollen in kurzem die Resultate wiedergegeben werden, die Zieler und Jansen bei ihren Untersuchungen mit dem Finsen licht erzielten, die sich auch in eingehender Weise mit den dabei auftretenden Zellveränderungen beschäftigten. $Z$ ieler belichtete das anämisierte Kaninchenohr mit einer FinsenR h ey n-Lampe 75 Minuten lang bei 15 bis $22 \mathrm{Amp}$. Stromstärke und 55 Volt Spannung, Jansen mit einem großen $\mathrm{F}$ insen-Konzentrationsapparat 60 Minuten bei $50 \mathrm{Amp}$. Stromstärke und 50 Volt Spannung. $\mathrm{Da}$ die Resultate beider Autoren annähernd übereinstimmen, so kann ich sie zusammenfassen:

Nach zwölf Stunden: Beginnende Zelldegeneration im Epithel, in Haarsäcken und Drüsen. Blutgefäße im Zustand der Stase. Beginnende Diapedese. Ein zellarmes Exsudat füllt die Spalträume ans, besonders stark an der unbelichteten Seite.

$\mathrm{Nach} 24$ Stunden: Teilweise bis völlige Nekrose der Epidermis und ibrer Anbangsgebilde. Thrombosierung der oberflächlichen Gefäße. Starke Diapedese. Wucherungsvorgänge des Epithels und der Bindegewebszellen auf der nicht belichteten Seite. Auch hier seröse Durchtränkung stärker als auf belichteter Fläche.

Nach drei Tagen: Nekrose des Epithels, der Haarbälge und Talgdrüsen. Thrombosierung der tiefen Gefäße, sogar einzelner der unbelichteten Seite. Starke reaktive entzündliche Vorgänge im Epithel und Bindegewebe der Randpartien, geringe im Zentrum.

Nach einer Woche: Tiefe Nekrose im bestrahlten Bezirk. Unter dem Knorpel bedentende Zellansammling. Knorpel ohne Kernund Protoplasmafärbnng. Deutliche entzündliche Regeneration im Zentrum, ganz bedeutend an der Peripherie und der nicht bestrahlten Seite.

$\mathrm{Nach} 14$ Tagen: Zentrum der Bestrablung mit einem Schorf bedeckt, völlige Regeneration des Epithels unterhalb desselben. Wucherung des Epithels in Zapfen. Stärkere Zellenneubildung in den oberen Cutisschichten, Rückgang derselben in der Tiefe (wenig Kernteilungen). Unbelichtete Seite stark hypertrophisch, zellreich.

Nach drei bis vier Wochen: Epidermis stark hypertrophisch. Das oberflächliche erheblich aufgelockerte Cutisgewebe in energischer 
entzündlicher Neubildung begriffen. Anordnung des neugebildeten Gewebes und der neuen Bindegewebsfasern in der Subcutis im Anschluß an die noch vorhandenen und nicht zu Grunde gegangenen kollagenen Fasern. Starke Wucherungen der Gefäßendothelien. Kerne und Protoplasma der Knorpelzellen mangelhaft gefärbt; an ihnen keine regenera. tiven Prozesse bemerkbar. An unbelichteter Fläche wieder im wesentlichen normale Verhältnisse.

Nach neun Wochen: Epidermis von normaler Dicke. Voll entwickelte Haare und Drüsen, Dichtes zell- und gefäBreiches Bindegewebe.

Welche Lichtquelle ist nun nach diesen Experimenten diejenige, welche eine größere Tiefenwirkung hervorruft, das Finsen licht oder das Licht der Quarzlampe?

Bevor ich diese Frage beantworte, möchte ich noch kurz auf die Ergebnisse der Quarzlampenbelichtung mit und ohne Methylenblauspülung eingehen. Schon makroskopisch fällt auf, daßohne Methylenblauzusatz zum Kühlwasser eine stärkere, zeitiger beginnende und später ablaufende Gewebszerstörung a uftritt als mit Methylenblau. Wie hochgradig die Nekrose ohne Methylenblau werden kann, geht daraus hervor, daß nach Abheilung des ganzen Prozesses bisweilen völlige Defekte im Gewebe zurückbleiben. Was den mikroskopischen Befund anlangt, so finden wirgleichfalls bei den Versuchen ohne Methylenblau die degenerativen Veränderungen bei weitem im Vordergrunde stehend. Schon nach 24 Stunden entwickelt sich eine deutliche Epithelnekrose, wäbrend mit Methylenblau regressive Prozesse nur erst wenig erkennbar sind. Nach drei Tagen ist ohne Methylenblau am Epithel eine deutliche Nekrose zu erkennen sowie Thrombosierung von Gefäßen und hochgradige Diapedesen, dagegen noch nirgends regenerative Prozesse. Mit Methylenblau tritt jedoch schon eine Zellemneubildung in dem stark ödematösen Bindegewebe der unbelichteten Seite hervor, während sowoll hier wie auf der bestrahlten Seite die degenerativen Veränderungen nicht sehr bedeutend sind. Nach einer. Woche hat sich bei beiden Versuchsanordnungen eine ausgedehnte Nekrose eingestellt. Sind jedoch in diesem Zeit- 
abschnitt die reaktiv-entzündlichen Vorgänge ohne Methylenblau erst im Entstehen begriffen, so ist mit Methylenblau die Zellproliferation im vollen Gange. Nach Verlauf von 14 Tagen hat sich bei Bestrahlung mit Methylenblau eine fast völlige Überhäutung vollzogen, an Stelle der untergegangenen Gewebselemente ist ein stark wucherndes Keimgewebe getreten, welches in den tiefen Schichten schon in der Zellneubildung nachgelassen hat. Ohne Methylenblau dagegen schreitet die Nekrose immer weiter vor, die Zellproduktion bleibt noch gering. So beobachtet man in der Tiefe der bestrahlten Partie noch keine regenerativen Prozesse, nur an den Rändern und der unbestrahlten Seite des Ohres hat sich gleichfalls ein Granulationsgewebe entwickelt. Nach drei Wochen ist die ohne Methylenblau bestrahlte Stelle zentral total nekrotisiert. Allerdings ersetzt ein in mächtiger Proliferation begriffenes Keimgewebe von der Peripherie her den Defekt. Mit Methylenblau findet man nach drei Wochen nichts mehr von degenerativen Veränderungen, auch die progressiven haben, abgesehen von der Knorpelzellwucherung in der Tiefe nachgelassen. In späteren Stadien (sieben Wochen) ist auch ohne Methylenblau eine völlige Regeneration der Gewebselemente eingetreten.

Der Hauptunterschied der Bestrahlung ohne und mit Methylenblauzusatz zum Kühlwasser ist also der, daß bei jener die destruktiven Prozesse, bei dieser die reaktiv-entzündlichen Vorgänge in den Vordergrund treten. Gerade diese aber sind die Träger einer aussichtsreichen Lichttherapie. Aus den Versuchen von $\mathrm{Z}$ iel ler und ron Jan s en mit dem F in s enlicht geht deutlich hervor, da $\beta$ das Fin s e nlicht ganz gewaltige regenerative Veränderungen sowohl in den oberen, wie besonders in den tiefen Gewebsschichten hervorzubringen im stande ist. Schon 24 Stunden nach der Bestrahlung treten die ersten Wucherungsvorgänge zu Tage. Diese nehmen im Bindegewebe, später auch im Epithel mehr und mehr zu, bis nach Ablauf von 14 Tagen wieder eine langsame Abnahme in der Proliferation zunächst in der Tiefe eintritt. Das zentrale noch von einem Schorf bedeckte Epithel ist zu dieser Zeit schon wieder 
hergestellt. Nach drei bis vier Wochen ist in den oberflächlichen Schichten noch immer eine erhebliche Zellenneubildung vorhanden, während im übrigen die Gewebselemente, abgesehen von einer Hyperplasie und Hypertrophie in toto zur Norm zurückgekehrt sind. Nach neun Wochen sind keine regenerativen Prozesse mehr vorhanden.

Wenn man diese Finsenwirkung mit der Reaktion vergleicht, die die Quarzlampe mit Methylenblauspülung denn nur so modifiziert kommt das Quarzlampenlicht in Betracht - an der Haut hervorbringt, so gelangt man zu dem Ergebnis, daß die Quarzlampe in ihrer Fähigkeit, in die Tiefe zu wirken, keineswegs zu unterschätzen ist. Die Wirkung des Finsenlichtes tritt allerdings bei weitem früher ein: Nekrose der Epidermis bereits nach 24 Stunden, zu gleicher Zeit bestehen schon entzündliche Veränderungen. Auf die Bestrahlung mit Methylenblau-Quecksilberdampflicht antwortet das Gewebe erst nach drei Tagen mit geringen regenerativen und gar erst nach fünf Tagen mit starken destruktiven Prozessen. Die Schädigung des Gewebes ist bei der Anwendung der Quarzlampe jedenfalls auch bei Methylenblauzusatzzum Kühlwasser eine stärkere und länger dauernde als bei der Finsenbelichtung, wie am deutlichten daraus hervorgeht, daß bei dieser die entzündlichen reaktiven Prozesse früher und energischer eintreten. Betrachtet man aber ein Bild nach Verlauf einer Woche, so ist ein großer Unterschied in der Reaktion nicht mehr vorhanden. Bei beiden Arten der Belichtung tritt am Albinokaninchenohr eine tiefe Nekrose des Epithels und seiner Anhangsgebilde sowie der Cutis mit ibren Gefäßen ein, die vielleicht bei Einwirkung der Quarzlampe viel erheblicher ist. Bei beiden ist aber ebenso eine lebhafte Gewebsneubildung im Gange. Und diese Proliferationsvorgänge, die bis nach Ablauf ron drei Wochen erheblich zunehmen, spielen sich hauptsächlich in der Tiefe ab. Hier wird das Bindegewebe zuerst wieder ersetzt. Hier tritt, nachdem ein kräftiges gefäßreiches Granulationsgewebe geschaffen worden ist, auch zuerst wieder Stillstand in der Regeneration ein. Für die bedeutende Tiefenwirkung der 
Quarzlampe spricht ferner die enorme Knorpelwucherung. Knorpelneubildungen wurden weder von $\mathrm{Zieler}$ noch von Jansen bei dem Finsenlicht beobachtet. Jener hat allerdings die Reaktion nicht länger verfolgt als bis vier Wochen nach der Bestrahlung, bei Bestrahlungen mit 20 bis $22 \mathrm{Amp}$. Stromstärke sogar nur 24 Tage, wobei allerdings mehrfach eine stark herabgesetzte Färbung des Knorpels und seiner Zellen bemerkt wurde. Jansen dagegen beobachtete einen Versuch bis nach Ablauf von neun Wochen. Er kommt bezüglich des Knorpels zu der Überzeugung, dal „Knorpel durch eine einstündige $\mathrm{Finsenbestrahlung} \mathrm{garnicht} \mathrm{zu} \mathrm{beeinflussen}$ zu sein scheint". Bei meinen sämtlichen Versuchen von 3 Wochen und darüber hinaus konnte ich aber eine Knorpelneubildung in der oben beschriebenen Weise feststellen, die um so stärker wurde, je weiter die Bestrahlungszeit zurücklag. Diese lebhafte Knorpelneubildung dürtte aber wohl hauptsächlich die Reaktion auf die bei Anwendung der Quarzlampe eintretende stärkere Gewebsschädigung sein; bei der Finsenbestrablung kommt es nicht leicht zu so starker Zerstörung.

Das Ergebnis meiner Untersuchungen ist folgendes:

Das Licht der Quarzlampe ruft olne Methylenblau bei direktem Auflegen auf die zu bestrahlende Stelle eine Nekrose hervor, die über das Maß dessen hinausgeht, was wir von einer therapeutischen Lichtquelle verlangen Die rege. nerativen Prozesse stellen sich sehr spät ein und vermögen unter Umständen die Lichtnekrose nicht wieder zu ersetzen (Gewebsdefekt). Die Schädigung des Gewebes ist eine so schwere-ähnlich wie Verbrennung oder Ätzung - daß sich das Gewebe nur sehr allmählich wieder erholt. Es trittdieanregende, die Regeneration befördernde, beziehentlich die die natürlichen Schutzmittel des Organismus freimachende Wirkung demgegenüber weit in den Hintergrund.

Das Licht der Quarzlampe mit Methylenblauzusatz zum Kühlwasser dagegen erzeugt eine der Finsenwirkung gleiche, oberflächliche, allerdings 
später eintretende Nekrose, weil die ganzoberflächlich wirkenden Strahlen nichtzur Wirkung kommen und so der schädigende Einfluß der Bestrahlung an der 0 berflächesich auch erst später zeigenkann, gleichzeitig mit der Wirkung in der Tiefe oder nachher. Die destruktiven und reaktiv entzündlichen Vorgänge in der Tiefe sind aber derart, daß sie den rom Finsenlicht gesetzten nicht wesentlich nachstehen, abgesehen daron, dab besonders dieregenerativenspäteraufreten, der schädigende Einfluß also stärker ist, als der die Heilung anregende. Meine Ergebnisse sind selbstrerständlich nicht ohne weiteres für die Frage zu verwerten, wie weit die Anwendung beim Menschen von gleichen Gesichtspunkten aus zu beurteilen ist. Zu beachten ist, daß die Dicke eines Kaninchenobres an der zur Bestrahlung gewählten Stelle nur $3 / 4-1 \mathrm{~mm}$ beträgt. Sie geben aber wohl im Verein mit den reichlich vorliegenden Untersuchungen über die Wirkung des Finsenlichtes brauchbare Vergleichspunkte. Jedenfalls hat die klinische Prüfung auch hiex ergeben, daß für Tiefenwirkung die Quarzlampe nur dann in Frage kommt, wenn die oberflächlich wirkenden Strahlen durch Methylenblauzusatz zum Kühlwasser ausgeschaltet werden.

Herrn Oberarzt Dr. Zieler spreche ich auch an dieser Stelle meinen verbindlichsten Dank aus für die Anregung zu dieser Arbeit und das liebenswürdige Interesse, welches er derselben entgegengebracht hat.

Die nach Abschluß meiner Versuche erschienene Arbeit von Stern nnd Hesse: Zur Tiefenwirkung des Quarzlampenlichtes (Dermatologische Zeitschrift 1907, Heft 8) beschäftigt sich hauptsächlich mit experimentellen und klinischen Untersuchungen an menschlichem Gewebe. St. und H. glauben, daß die Quarzlampe für Lupus und andere tiefer greifende Erkrankungen eine ebenso günstige Wirkung wie das $F$ insen licht nicht entfalten könne. Sie beobachteten bei 10 Minuten langen Belichtungen mit und ohne Methylenblauspülung eine starke, bereits nach 48 Stunden auftretende Epithelnekrose, während ein der 
Finsenbelichtung entsprechendes Ödem nicht auftrat. Dieselben Resultate wurdon auch gezeitigt bei Versuchen mit Albinokaninchen, die in derselben Weise angestellt wurden, wie die von mir beschriebenen. Ich kann diesen Tierexperimenten gegenüber nur betonen, daß icb, was die Belichtungen besonders mit Methylenblauspülung anlangt, nicht die gleichen Ergebnisse geh: bt habe. Wenn bereits nach 48 Stunden ein makroskopisch sehr deutlicher Schorf auftrat, so war das $\mathrm{Obr}$ nicht genügend (in der von $\mathrm{Finsen}$ angegebenen Weise) durch Kompression anämisiert bzw. nicht genïgend fest an das Quarzfenster angepreßt. Ferner stellte ich bei sämtlichen einwandsfreien Versuchen mit Methylenblau ein deutliches ödem, welches besonders stark auf der unbestrahlten Seite war, fest, analog der Finsenwirkung, wenn auch auf der bestrahlten Seite viel schwächer ausgebildet. Da bei Bestrahlung ohne Methylenblau dieses entzündliche Ödem bedeutend geringer ist, a d der bestrahlten Seite sogar zunächst völlig fehlt, und die Nekrose bei weitem rascher auftritt und tiefer greift, so müssen wir annehmen, daß durch Absorption der ganz kurzwelligen ultravioletten Strahlen (durch Methylenblau) diese starke oberflächliche Nekrose nicht oder nur gering statthaben und somit dieweniger nekrotisierende Wirkung in der Tiefe besser und energischer eintreten kann, d. h, es treten die schädigenden Wirkungen des Lichtes zurück und es kommen die durch die Belichtung angeregte entzündliche Hyperämi"e undihrebeilenden Kräfte mehr zur Geltung. 